\title{
EVOLUTIONARY POPULATION SYNTHESIS MODELS OF PRIMEVAL GALAXIES: A CRITICAL APPRAISAL
}

\author{
A. BUZZONI \\ Osservatorio Astronomico di Brera \\ Via Bianchi, 4623807 - Merate (Lc), Italy
}

\section{Introduction}

A recognized problem when searching for primeval galaxies at cosmological distances is a definition of a firm selection criterion (alternative to a direct but extremely time-demanding measure of $z$ ) to single out high-redshift candidates from the plethora of other more or less peculiar objects (i.e. AGN, starburst galaxies etc.) at lower distances.

The case of the HST "Deep Field" (HDF) observations (Williams et al. 1996) is especially relevant in this regard as galaxies up to $z=3.2$ have been detected in the field (Steidel et al. 1996).

Taking advantage of the selective effect on galaxy apparent colors due to foreground $L_{\alpha}$ absorption from intergalactic Hydrogen clouds (the socalled $L_{\alpha}$ forest), Madau et al. (1996) have been able to select star-forming galaxies in the HDF up to a fiducial redshift of $z \sim 4.5$.

This method complements the more direct empirical approach of Lanzetta et al. (1996) who probed galaxy redshift by fitting observed colors with spectra of local templates of appropriate morphological type. The latter procedure is however prone to a subtle internal inconsistency since it implicitly neglects any evolutionary effect.

On the other hand, the main drawback of Madau's et al. method resides in its selection bias toward a special class of candidates (the so-called "dropout" objects). Clearly, this makes difficult to assess how representative is the selected subsample of the whole galaxy population at the relevant redshift.

In this framework, a theoretical approach relying on evolutionary population synthesis models could help refining the search criteria in deep galaxy surveys on the basis of a better knowledge of the expected apparent photometric properties of high-redshift objects. The following is a brief discussion 
reviewing some relevant aspects of the question in order to allow a more critical appraisal to primeval galaxy recognition.

\section{Global Energetics in Model Stellar Populations}

It is especially convenient to consider a model galaxy in terms of its composing simple stellar populations (SSPs), that is of single generations of coeval stars with fixed distinctive parameters like metallicity, initial mass function (IMF) etc.

A basic problem when tracking SSP evolution deals with a correct evaluation of Main Sequence (MS) and Post-Main Sequence relative contribution to the SSP total luminosity. From the theoretical and operational point of view in fact these two main building blocks are managed in a substantially different way.

While Post-MS star distribution is simply proportional to the lifetime duration in each isochrone bin (so that $n_{i} / n_{j}=t_{i} / t_{j}, \forall i, j$ ), MS number counts are on the contrary modulated by the IMF (namely $n_{i} \propto M_{i}^{-s}$ for a standard Salpeter power law). We need therefore to know how to scale Post-MS as a whole with respect to the MS component in the model SSP.

Clearly, a simple match by grafting contiguous isochrone bins at the MS top and at the Post-MS bottom cannot be a safe solution in this regard as any uncertainty and numerical "noise" in the evaluation of both extrema would accordingly reverberate into a magnified scatter in the contribution of each SSP building block.

Rather than relying on such a differential normalization procedure, one could take advantage of an integral approach featuring SSP global properties. This can be done via the so-called "Fuel Consumption Theorem" (FCT) (Renzini and Buzzoni 1986) dealing with SSP energetics. Starting from the general relation in Renzini and Buzzoni (1986, their eq. 14), it is possible to state the theorem as follows:

$$
\frac{L_{P M S}}{L_{\text {tot }}}=\mathcal{B} \times \text { Fuel }=(1.76 \pm 0.4) m_{H}
$$

This simply relates SSP Post-MS luminosity to the total amount of fuel spent in Post-MS evolution by stars of mass $M_{T O}$ leaving the MS Turn Off point.

In the equation, the normalization factor $\mathcal{B}$ is the so-called "specific evolutionary flux"; it turns to be about $\mathcal{B}=1.7 \pm 0.410^{-11}\left[\mathrm{~L}_{\odot}^{-1} \mathrm{yr}^{-1}\right]$ (Buzzoni 1989). The term $m_{H}$ is the exhausted fuel expressed in Hydrogenequivalent solar masses. This quantity is a straightforward output of stellar evolution theory. 


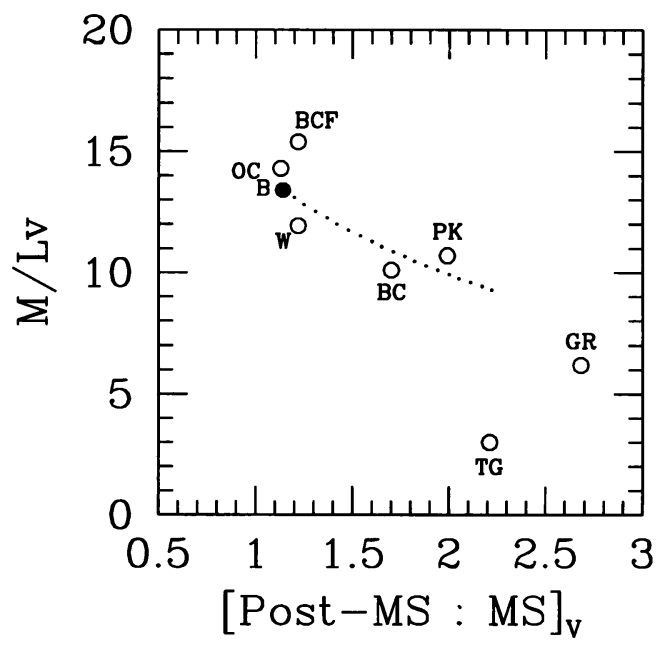

Figure 1. Comparison of template model ellipticals from different theoretical codes for population synthesis. Displayed are the predicted $M / L_{t o t}$ ratio and $L_{P M S} / L_{M S}$ $V$-luminosity partition from the models by O'Connell (1976) [OC], Tinsley and Gunn (1976) [TG], Pickles (1985) [PK], Guiderdoni and Rocca-Volmerange (1987) [GR], Bruzual and Charlot (1993) [BC], Worthey (1994) [W], Bressan et al. (1994) [BCF], and Buzzoni (1995) [B]. The effect of artificially doubling Post-MS contribution (then relaxing FCT prescriptions) is displayed for Buzzoni's models by the dotted line.

It might be interesting to check whether FCT prescriptions are consistently met by current theoretical synthesis codes, some of them extensively adopted for cosmological studies. Following the original discussion in Buzzoni (1995), in Fig. 1 we have compared template models for present-day elliptical galaxies according to the different theoretical sources.

A striking evidence from the figure is that model ellipticals by Tinsley and Gunn (1976), Pickles (1985), Guiderdoni and Rocca-Volmerange (1987), and Bruzual and Charlot (1993) do not fully comply with the SSP energetic constraint in the sense that all of them appear to be sensibly overestimating Post-MS contribution.

Focussing for example on the Bruzual and Charlot (1993) model, (Fig. 2, left panel) one can appreciate a noticeable scatter, of the order of $\pm 50 \%$, with respect to Buzzoni's (1995) calculations. Note in addition that PostMS excess in the model at older ages abruptly reverses for $t<10 \mathrm{Gyr}$. As far as cosmological predictions are concerned, diverging conclusions would be achieved, as shown in Fig. 2 (right panel). In particular, while Bruzual and Charlot would predict a redder apparent $B-V$ for low-redshift ellipticals with respect to Buzzoni's model, colors would then turn to be much bluer with increasing $z$. 


$$
\left(\mathrm{H}_{\mathrm{o}}, \mathrm{q}_{\mathrm{o}}, \mathrm{z}_{\mathrm{f}}\right)=(50,0,30)
$$
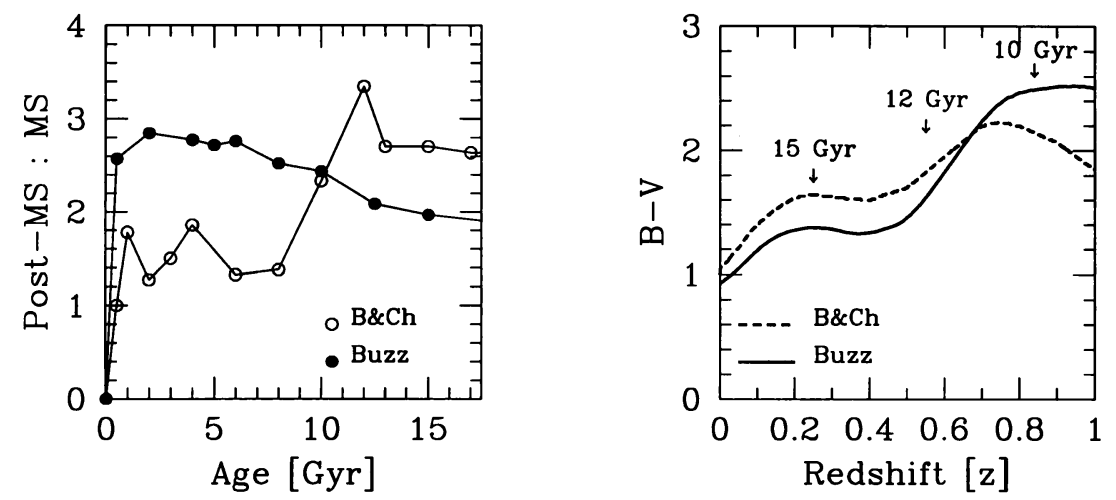

Figure 2. (left panel) - Bolometric evolution of template elliptical galaxies from Bruzual and Charlot (1993) (०) and Buzzoni (1995) (•). For a 15 Gyr SSP, $m_{H}$ in eq. (1) is of the order of $0.35 M_{\odot}$ (Renzini and Buzzoni 1986) so that $L_{P M S} / L_{M S} \simeq 2$ in bolometric, according to FCT prescriptions.

(right panel) - Predicted apparent colors vs. redshift in a $\left(H_{o}, q_{o}\right)=(50 \mathrm{~km} / \mathrm{sec} / \mathrm{Mpc}, 0)$ Universe. Absolute age of ellipticals is labeled along the curves assuming a redshift of galaxy formation $z_{f}=30$.

\section{Galaxy Back-in-time Evolution}

A new complete set of model galaxies of different morphological type, spanning the whole Hubble sequence, has been produced via a semi-analytical approach relying on SSP theory (Buzzoni 1997).

Each model consists of a spheroid component (that is a bulge+halo system) and a disk. Appropriate luminosity partition among the different galaxy subsystems with changing morphology, as well as adopted star formation history have been tuned by probing galaxy evolutionary status as observed at present time (cf. Buzzoni 1997 for a full discussion of the theoretical details). A sample of our results is displayed in Fig. 3.

Two major features seem to characterize primeval galaxy evolution:

i) all systems currently displaying even a marginal luminosity contribution from the bulge (like for example in Sd spirals) have been largely dominated by the nuclear emission early in the past;

ii) for a wide range of galaxy star formation histories, all morphological types tend to evolve back in time toward (restframe) bluer colors.

The natural conclusion of our brief analysis is therefore that primeval galaxies would look like very nucleated blue objects.

According to Fig. 4 (left panel), there are at least two main sources of bias that could induce (opposite) misleading interpretations of high-redshift 


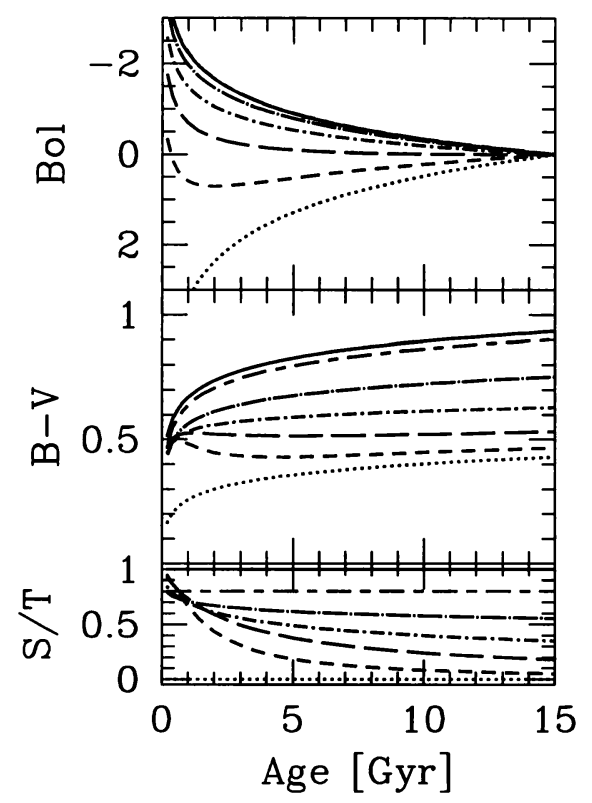

Figure 3. Theoretical evolution of template model galaxies of different morphological type according to Buzzoni (1997). The following types are considered in the three panels: E (solid line), S0 (dot long-dashed), Sa (dot short-dashed), Sb (long-dashed), Sc (short-dashed), Im (dotted). Upper panel reports bolometric evolution by normalizing model luminosity at $15 \mathrm{Gyr}$ absolute age; middle panel shows the restframe $B-V$ color; in the lower panel, the galaxy morphological parameter $S / T$ measures the actual relative fraction of total bolometric luminosity provided by the spheroid (mostly bulge) component. Every model (excepting Im galaxies) ends up to be bulge-dominated early in the past.

data when neglecting evolutionary effects.

i) As far as only galaxy apparent colors are taken into account (that is with no hints about morphology) one would be detecting a substantial deficiency of (intrinsically) red objects at high redshift, and an apparent excess of blue (supposedly "active") galaxies. This might account for instance for Madau's (1997) results by matching HDF and the Canada-France redshift survey (Lilly et al. (1996).

ii) Conversely, if we only rely on the morphological features of distant galaxies with no information about their colors, then one would lead to conclude that early-type systems were dominating in the early Universe while spirals would ostensibly be missing at high redshift. An example in this sense is the HDF morphology study by van den Bergh et al. (1996). As shown in Fig. 4 (right panel), compared with low-redshift morphology distribution, in the HDF there is a lack of Sb-Sc spirals in favor of an important excess of bulge-dominated systems ( $\mathrm{E}$ and Sa spirals). 

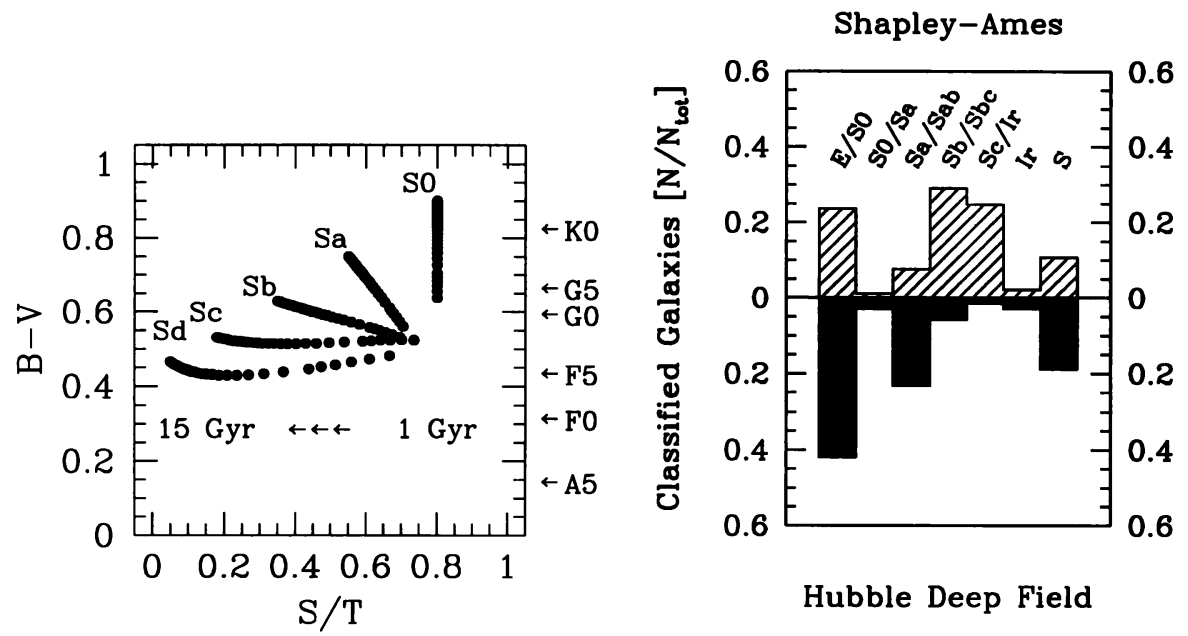

Figure 4. (left panel) - Restframe $B-V$ color for model galaxies of different morphological type according to Buzzoni (1997). Evolution is computed for absolute age between 1 and 15 Gyr, as labeled. Typical spectral types for stars of similar relevant color are marked in the $y$-axis right scale. The $S / T$ parameter refers to the actual bolometric contribution of galaxy bulge. $S / T \rightarrow 1$ for very nucleated objects.

(right panel) - Morphology distribution of galaxies in the HDF compared with the local Shapley-Ames sample, according to van den Bergh et al. (1996). A biased lack of Sb-Sc galaxies seems to occur in the HDF in favor of E-Sa systems.

\section{References}

Bressan, A., Chiosi, C., and Fagotto, F. 1994, ApJS, 94, 63

Bruzual, G., and Charlot, S. 1993, ApJ, 405, 538

Buzzoni, A. 1989, ApJS, 71, 817

Buzzoni, A. 1995, ApJS, 98, 69

Buzzoni, A. 1997, ApJ submitted

(see also http:\|www.merate.mi.astro.it $\sim$ eps $\backslash$ home.html)

Guiderdoni, B., and Rocca-Volmerange, B. 1987, $A \& A$, 186, 1

Lanzetta, K.M., Yahil, A., and Fernández-Soto, A. 1996, Nature, 381, 759

Lilly, S.J., Le Fèvre, O., Hammer, F., and Crampton, D. 1996, ApJL, 460, L1

Madau, P. 1997 in "The Hubble Deep Field", STScI Symp Series, eds. M. Livio, S.M. Fall and P. Madau (STScI: Baltimore) in press

Madau, P., Ferguson, H.C., Dickinson, M.E., Giavalisco, M., Steidel, C.C. and Fruchter, A. 1996, MNRAS, 283, 1388

O'Connell, R.W. 1976, $A p J$, 206, 370

Pickles, A.J. 1985, ApJ, 296, 340

Renzini, A., and Buzzoni, A. 1986 in "Spectral Evolution of Galaxies", eds. C. Chiosi and A. Renzini (Dordrecht: Reidel) p. 195

Steidel, C.C., Giavalisco, M., Dickinson, M.E., and Adelberger, K.L. 1996, AJ, 112, 352

Tinsley, B.M., and Gunn, J.E. 1976, $A p J$, 203, 52

van den Bergh, S., Abraham, R.G., Ellis, R.S., Nial, R.T., Santiago, B.X., and Glazebrook, K.G. 1996, $A J$, 112, 359

Williams, R.E. et al. 1996, $A J, \mathbf{1 1 2}, 1335$

Worthey, G. 1994, ApJS, 95, 107 